K.A. van der Hucht, G. Koenigsberger \& P.R.J. Eenens, eds.

\title{
Wolf-Rayet galaxies: the past is prologue
}

\author{
Peter S. Conti \\ JILA, University of Colorado, Boulder, CO 80309-0440, USA
}

\begin{abstract}
Wolf-Rayet galaxies are those starburst systems in which the presence of Wolf-Rayet stars is inferred from the appearance of broad emission lines, notably $\lambda 4686$ HeII. The ages of the bursts are only a few Myr when WR stars are present. Many (all?) of these starburst galaxies are interacting or merging systems. This talk will review the methods used to estimate the hot star populations in these galaxies, with emphasis on optical spectrophotometry. It will be important to keep in mind that the discovery of WR stars in starburst galaxies is highly dependent on spectral resolution, signal to noise, and the local properties of the object since one is searching for broad but weak emission lines superimposed upon a galaxy continuum. A brief comparison of the inferred numbers of O-type and WR stars in a galaxy, compared to the predictions of the most recent burst models, reveals good agreement in most cases. The exceptions might be understood in terms of geometric dilution (i.e., the stars and the ionized nebular gas are not co-spatial).
\end{abstract}

\section{Introduction}

Wolf-Rayet galaxies (Conti 1991) are defined as those galaxies (or parts thereof) with broad emission lines of highly excited ions, notably $\lambda 4686 \mathrm{HeII}$. All of these galaxies also show narrow nebular recombination emission lines from thermally excited gas, in particular the Balmer series. This larger class of galaxies are sometimes referred to as ' $\mathrm{H}$ II' galaxies, emission line, or starburst galaxies. The presence of nebular recombination lines indicates that hot, luminous stars are present, primarily O-type (although the WR stars also contribute). Similarly to WR stars, WR galaxies show the presence of WN and WC subtypes, the representative lines being $\lambda 4686 \mathrm{He}$ II and $\lambda 4640 \mathrm{~N}$ III for the former subtype and $\lambda 5808 \mathrm{C}$ IV and $\lambda 5696 \mathrm{C}$ III for the latter. With one exception (NGC 1365 - which may only be a detection problem), all WR galaxies contain WN stars and a little less than half, WC types (Schaerer et al. 1999).

The statistics of WR galaxies have recently been greatly advanced by the new survey of Schaerer et al. (1999 - SC; see also Contini 1999). The first WR galaxy, He2-10, was discovered by Allen et al. in 1976; the first WR catalogue (Conti 1991) listed 37 objects; the new compilation (SC) has 126. These numbers indicate a doubling time for the discovery of new WR galaxies of 3.5 years. Clearly this rate cannot be maintained for too much longer, but it is fun while it lasts.

SC discuss the presence of narrow (nebular) $\lambda 4686 \mathrm{HeII}$, from very highly excited gas, whose origin is not fully understood but must come from very hot stars. They find 47 (37\% of) WR galaxies with this feature; those with nebular $\lambda 4686 \mathrm{He}$ II and WC stars number $31(66 \%)$; there are only $9(28 \%)$ with narrow 
$\lambda 4686$ but no WC stars. These data suggest that WC stars play a role in producing the nebular He II feature in some cases.

Most of the spectroscopy of WR galaxies has been in the optical. It is instructive to examine and compare the spectra of He2-10 in Allen et al. (1976), Vacca \& Conti (1992), and most recently by Schaerer et al. (1999). In the first paper a broad emission feature at $\lambda 4660$ is seen and identified as having contributions from both $\mathrm{N}$ III $(\lambda 4640)$ and He II $(\lambda 4686)$. These features are also found in Vacca \& Conti with higher spectral resolution but similar signal to noise. The Schaerer et al. spectrum, much improved over the previous two, shows the same broad features but with the additional presence of a narrow nebular feature due to [FeIII] at $\lambda 4658$. This line, along with emission lines of [ArIv] at $\lambda \lambda 4711,4740$ are found in some WR galaxies and can complicate the interpretation of the broad emission features unless relatively high spectral resolution is available.

There is sparse published spectroscopy of WR galaxies in the UV with HST. The $\lambda 1640 \mathrm{He}$ II line has been detected in NGC 4214 (Leitherer et al. 1996) and in NGC 1741 (Conti et al. 1996). The N IV semi-forbidden emission feature at $\lambda 1483$ is present in He2-10 (Johnson et al. 1999).

\section{Observations of Wolf-Rayet galaxies}

\subsection{Spectrophotometry}

The overall spectrum of a galaxy is a spatial integration over the sky through an entrance aperture or spectroscopic slit. The UV and optical radiation from WR (and emission line) galaxies are provided by stars, not only from the starburst itself, but also by the background, pre-existing object. The stellar content of the burst includes the prominent massive and hot OB types, but also those of lower mass, perhaps even down to a solar mass (although the lower mass cutoff is not yet well established). The galaxy is typically older and may have no massive OB stars present. The contributions of the various stellar populations are highly wavelength dependent.

Optical. The continuum mostly arises from B and A type stars in the starburst, as may be inferred from their predicted luminosity contribution to a burst using a normal IMF slope ('Salpeter'). This is confirmed by the presence of a narrow $\mathrm{K}$ line and the upper Balmer series lines in absorption (Vacca \& Conti 1992), also signatures of these spectral types. The nebular emission from the excited interstellar gas is produced by the O (+WR) stars. WR stars are the source of the broad emission lines. In typical WR stars these lines have equivalent widths of a few hundred $\AA$; in WR galaxies the strengths are a few $\AA$. Thus the continuum dilution from other stars in the burst is about 2 dex.

Ultraviolet. The $\mathrm{OB}(+\mathrm{WR})$ stars produce the continuum; the A and later type stars have relatively little contribution. Absorption lines due to $\mathrm{O}$ stars have been found, but the dominant spectroscopic features are the P-Cygni profile wind lines. These, too, are from the hot, luminous OB types. Broad emission lines are due to the WR stars.

Infrared. The continuum at IR and longer wavelengths can be dominated by local dust emission, for example in 'luminous IR galaxies' (e.g., Joseph 1999). The strength of this emission is due to the most luminous stars, those of type 
OB; WR stars may contribute but only marginally. I don't recall seeing any IR spectroscopy of WR galaxies showing either absorption or broad emission features analogous to those found in the optical. A complicating factor may be that red supergiants in the burst or the background galaxy can dominate the radiation at NIR wavelengths. Nebular emission lines are found in the IR; as in the optical, these are thermal lines excited by the OB (+WR) stars in the starburst. A recent analysis of such lines in NGC 5253 has been carried out by Crowther et al. (1998).

\subsection{Analyses of hot star populations}

I will briefly review the methods used to analyze the hot star populations in WR galaxies; these have also been utilized in other galaxies with emission lines. The first three methods are distance dependent but give the numbers of hot stars.

Nebular: Lyman Continuum photons (Lyc). The line fluxes in the Balmer lines (e.g., $\mathrm{H} \beta$ ) are used to estimate the number of Lyc photons emitted by the gas using photoionization modeling (e.g., Stasinska 1999) and the population estimate of the stars is thus derived from indirect measurements.

An important assumption is that the ionized gas and the stars are cospatial, but it is usually the case that the gas volume is larger than the hot star volume. Although one will measure the integrated spectra of all the stars seen in projection in the slit this may well be smaller than the area of the emitting gas. I will call such a situation a geometrical dilution as there is a mismatch between the stellar contribution and the nebular one. I will show later that this effect might be quite important in some cases.

Even if the gas and stars are co-spatial, one might have a continuum dilution from the background. The typical models of starbursts take into account the continuum dilution of the burst as it controls the observed equivalent widths of nebular lines such as $\mathrm{H} \beta$ and WR emission lines such as $\lambda 4686 \mathrm{HeII}$, but the galaxy itself may contribute. I've been concerned with this issue for some time and will address it below.

Further constraints are: ( $i$ ) The exciting gas is ionization bounded (i.e., no leakage of Lyc photons); (ii) There is negligible absorption of Lyc photons by the dust. The procedure is then to measure the (say) $\mathrm{H} \beta$ flux, correct for reddening and thru well known nebular line calculations obtain the numbers of exciting stars given the convenient calibration that one O7V 'equivalent' star (e.g., Vacca 1994) produces $10^{49}$ Lyc photons per second. This can be converted to the 'numbers' of $\mathrm{O}$ stars by adopting an IMF slope and an upper mass cutoff $\left(M_{u p}\right)$, accounting for the abundance dependence and the contribution of WR stars, using the updated prescriptions of Schaerer \& Vacca (1998 -SV).

Broad emission lines. The extinction corrected flux of WR emission lines gives the number of WR stars. The calibration for individual WR stars is, for example, a luminosity for $\lambda 4686 \mathrm{He}$ II in WNL stars of $5.2 \times 10^{36} \mathrm{erg} \mathrm{s}^{-1}$ according to SV; other lines may also be utilized. The accuracy depends both on the measurement of the lines in the WR galaxies, and the calibration of WR spectral subtypes. The latter numbers may only be good to a factor of 2 .

Continuum - UV. In this spectral region most of the radiation arises from the hot, luminous stars. Measure the monochromatic flux (at some convenient 
wavelength free of lines) and correct for reddening (and adopt an extinction law). From models one can obtain a 'calibration' of the monochromatic flux at the appropriate wavelength that additionally depends on the age. With an IMF slope and a $M_{u p}$ one obtains the 'numbers' of OB (+WR) stars. This is a very powerful and direct method which, combined with those above, leads to accurate estimates of the hot star populations. Aside from highly redshifted starburst galaxies, it has only been applied in detail in a handful of cases, but more can be expected with further HST observations.

P-Cygni wind profiles - $U V$. This method, which is distance independent, utilizes measurements of the normalized profiles of the UV wind lines. These are then analyzed in a 'spectral synthesis' procedure (Leitherer et al. 1995) to estimate the age of the burst (needed above). One also obtains some constraint on the IMF slope and $M_{u p}$ (e.g., Leitherer \& Heckman 1995).

Absorption lines. To the best of my knowledge, these have not yet been utilized although they could, in principle, be included in the modeling predictions.

\section{Starburst model spectral synthesis predictions}

Leitherer (1999) has reviewed and compared the various starburst model predictions for WR galaxies which in some cases vary dramatically among different authors. Here I wish to concentrate on the connection with the observations utilizing the Geneva Group models, in this case taken from SV and Schaerer et al. (1999 - see also Schaerer 1999). I will utilize only optical spectroscopic data from Vacca \& Conti (1992) and Schaerer et al., mainly for consistency; other authors have not always measured the $\lambda 4686 \mathrm{He}$ II emission line strength separately from the blending lines of $\mathrm{N}$ III and the overlapping forbidden features.

The predicted equivalent widths of WR lines in starburst galaxies depend somewhat on the metal abundance, being stronger and spread over a longer time interval for larger $Z$. The sample of WR galaxies noted above have typical $Z$ values of 0.008 to 0.004 ; in Figure 13 of SV the He II and C IV lines are predicted to have line strengths of a few $\AA$ between the ages of 3 to $6 \mathrm{Myr}$. Do the starburst galaxies with WR stars have inferred ages within these ranges? To answer this question, I use Figure 7 of SV, which predicts the age as a monotonic (but abundance dependent) function of $\mathrm{H} \beta$ equivalent width; as the burst ages the emission lines decrease in strength. I take the $\mathrm{H} \beta$ equivalent width from the WR galaxy sample of Vacca \& Conti (1992). Some of their galaxies do not show WR features and these are plotted with a different symbol in Figure 1.

The WR galaxies of Figure 1 do indeed have inferred ages of 3-6 Myr, as predicted, a nice confirmation of the theory. Unfortunately, several starburst galaxies without WR stars have similar implied ages (although a few are outside the time limits). How can one understand the presence of starburst galaxies with ages of 3-6 Myr but without detected WR stars? This could be partly explained by continuum dilution by the background galaxy; a factor 2 dilution would make the broad emission features unobservable (and while also diluting the $\mathrm{H} \beta$ equivalent width this only lengthens the inferred age a little). A superficial examination of optical images of the non WR starburst galaxies did not reveal any obvious differences in background dilution among the WR galaxies but detailed analyses would have to be performed, preferably with HST-WFPC. 


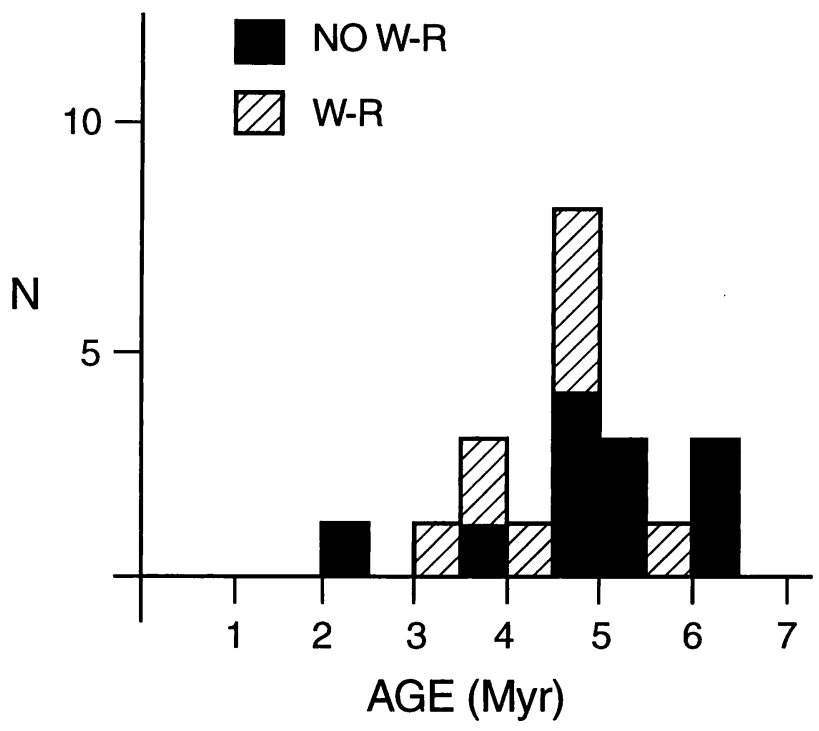

Figure 1. Histogram showing the distribution of WR (and non WR) galaxies with age (inferred from the $\mathrm{H} \beta$ equivalent widths).

Generally, starburst galaxies older or younger than 3-6 Myr would not be expected to have WR stars present.

What now of the predicted line strengths of $\lambda 4686$ He II as compared to $\mathrm{H} \beta$ for WR galaxies? I will adapt Figure 13 of Schaerer et al. (1999) and plot the data as shown in Figure 2. The predictions of the so called standard model includes the effect of 'normal' Of stars; the inclusion of very luminous, hydrogen burning WN stars like those found in R 136 is also given. Four objects are in common between Vacca \& Conti (1992) and Schaerer et al. (1999) in which there is good agreement in the measured $\mathrm{H} \beta$ equivalent widths, but in two or three galaxies there is a substantial difference between the $\lambda 4686 \mathrm{He}$ II values. I have no ready explanation for this, although I would expect the more recent paper, being from higher quality spectroscopy, would be more accurate. In any event, with three (or possibly four) exceptions, most of the observed galaxy values are close to the predictions of Schaerer et al. This gives us considerable confidence in the procedure and we can infer that the continuum dilution is small (i.e., little contribution from the underlying galaxy).

The three (or four) outliers of Figure 2 all have considerably stronger $\lambda 4686$ than would be predicted from their measured $\mathrm{H} \beta$ equivalent widths. This cannot be a continuum dilution problem as it would affect both lines equally. It is possible that the $\mathrm{H} \beta$ is weaker than expected due to leakage of Lyc photons from the star burst region and/or dust absorption. Notice the two ' $\mathrm{X}$ '-values that are close to the outliers and one that is on the predicted relationship. I will discuss these points in the next section, as they bear upon the galaxies with anomalously strong $\lambda 4686$ He II or weak $\mathrm{H} \beta$. 
ADAPTED FROM SCHAERER et al. 1998

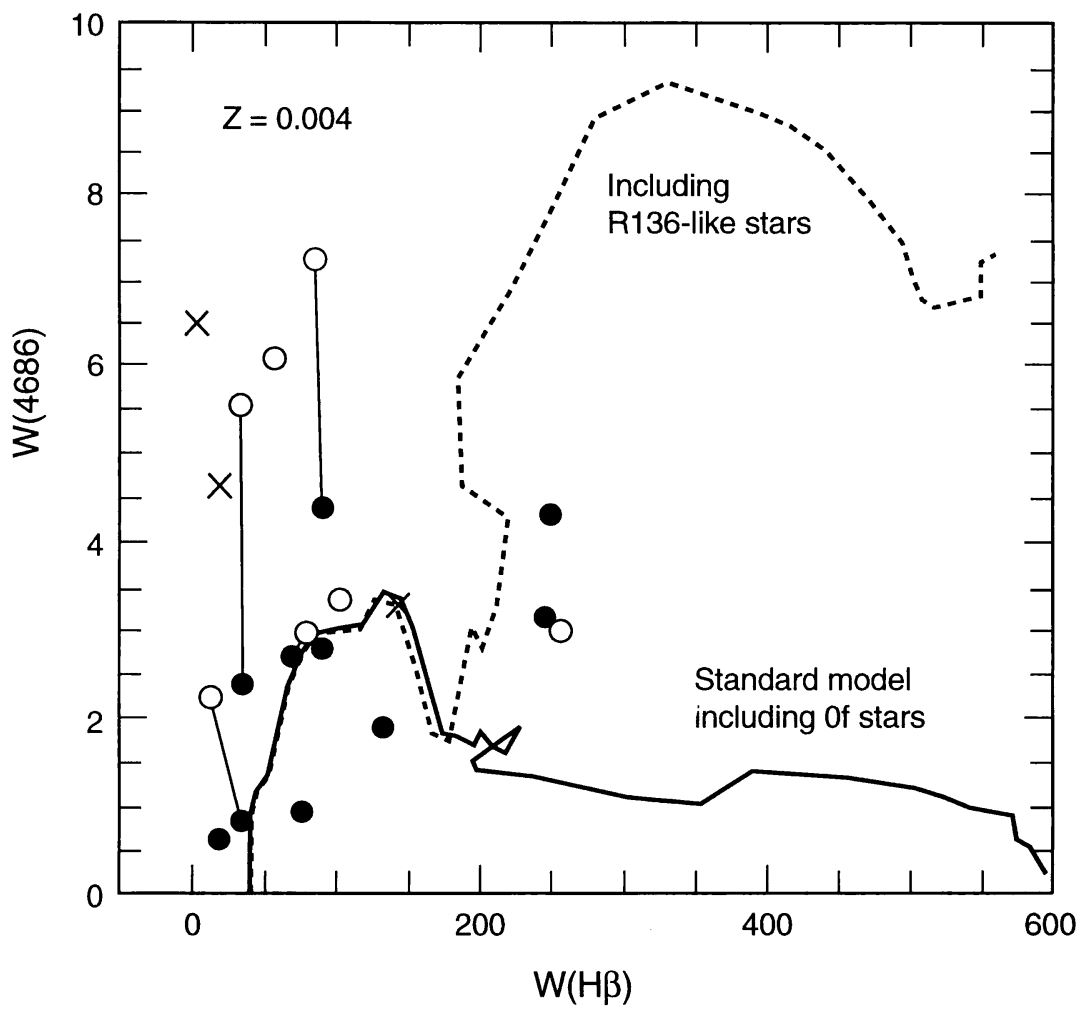

Figure 2. Predicted (solid lines) dependence of $\lambda 4686$ with $\mathrm{H} \beta$ for WR galaxies; the filled symbols are the galaxies of Vacca \& Conti (1992), the open symbols data from Schaerer et al. Thin lines connect data from the same objects. The X symbols are discussed in $\S 4$.

\section{Geometric dilution}

A local example of the substantially different volume (area) distributions of exciting stars and ionized gas can potentially help us understand Figure 2. Mark Phillips and I acquired a number of optical spectrophotometric drift scans of the 30 Dor region. These are obtained by utilizing a long slit and letting the telescope drift at a rate such that an appropriate area is traced out (similar to the procedure for UV spectroscopy with the IUE telescope and reported by Vacca et al. 1995). Figure 3 illustrates the areas sampled.

$\mathrm{W}$. Vacca and T. Ramond have reduced and made a preliminary analyses of the integrated spectra of 30 Doradus for the various areas (volumes) indicated by Figure 3. I show in Figure 4 their extinction corrected spectra. The continuum levels differ by a factor two or so, whereas if one uses the ratio of the areas one would have expected a factor 9 . This tells us formally that the stars are centrally concentrated, which is also obvious from the image. The strength of the $\lambda 4686$ He II emission feature is similar from region to region and correctly 


\section{Doradus}

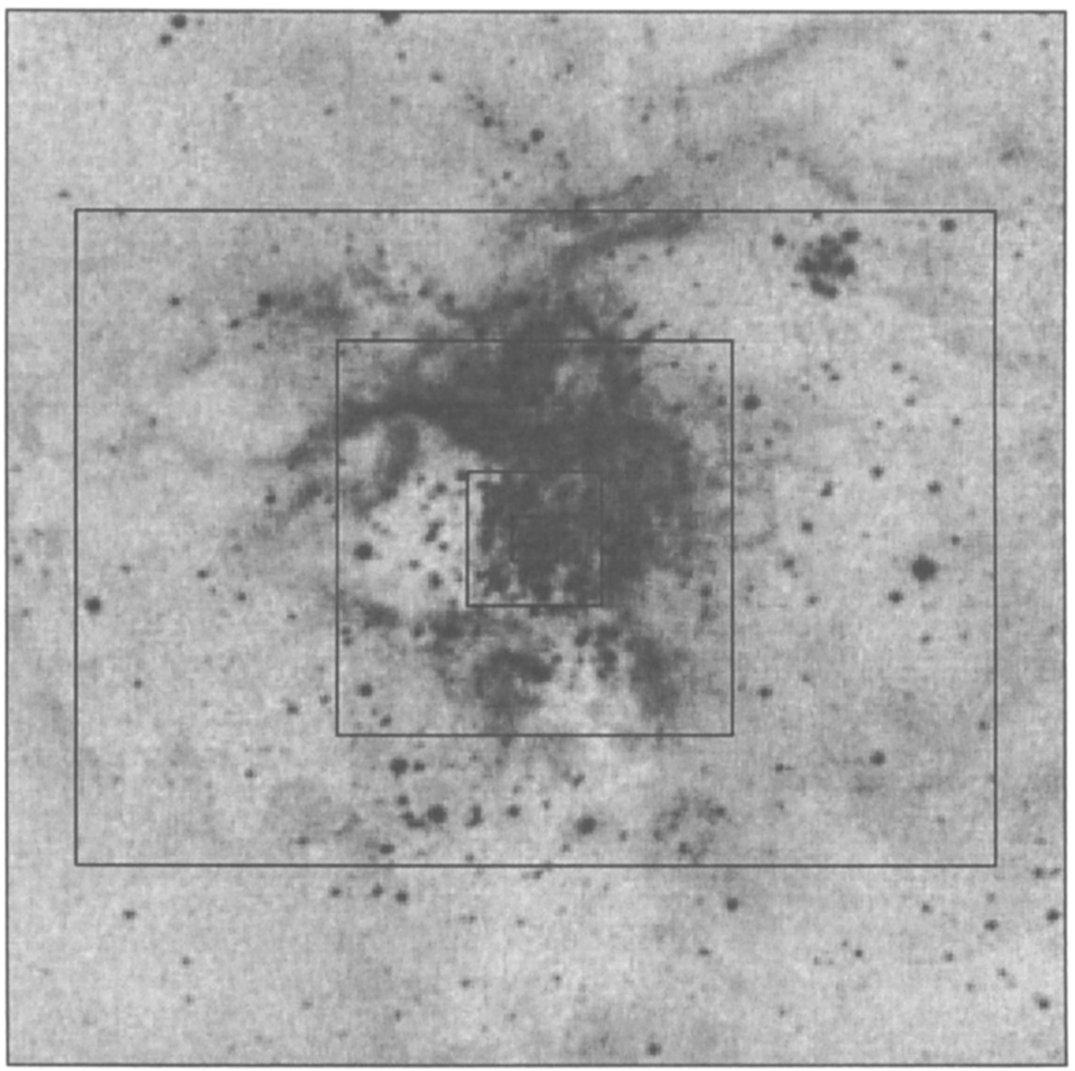

Figure 3. Optical image of the 30 Doradus region with the drift scan areas superimposed. The areas correspond to $20^{\prime \prime} \times 20^{\prime \prime}$ (fully containing R 136), $1^{\prime} \times 1^{\prime}, 3^{\prime} \times 3^{\prime}$ and $5^{\prime} \times 7^{\prime}$. About half of the exciting stars are within the smallest area, but clearly considerable ionized gas is found outside this region, even exceeding the largest area.

predicts the number of WN stars obtained by actual count, some 20 in number; most of these are in R 136 .

The measured $\mathrm{H} \beta$ and $\lambda 4686$ He II equivalent widths (kindly provided by Bill Vacca) are plotted in Figure 2 as the ' $\mathrm{Xs}$ '; the weakest $\mathrm{H} \beta$ s correspond to the smallest areas of Figure 3 . However, the $3^{\prime} \times 3^{\prime}$ area ' $\mathrm{X}$ ' position agrees reasonably well with the predictions of Schaerer et al. (1999). In other words, using the $3^{\prime} \times 3^{\prime}$ aperture we obtain rough agreement between observation and predictions. Even this area does not include all the ionized gas. Correcting for this effect might roughly double the $\mathrm{H} \beta$ equivalent width, thus placing the observed ' $\mathrm{X}$ ' in Figure 2 on the predicted line for 'R 136 stars' (as, of course, it should).

We may conclude that the discrepancy of $\lambda 4686$ and $\mathrm{H} \beta$ values of several WR galaxies in Figure 3 might be explainable by geometric dilution: considerable 


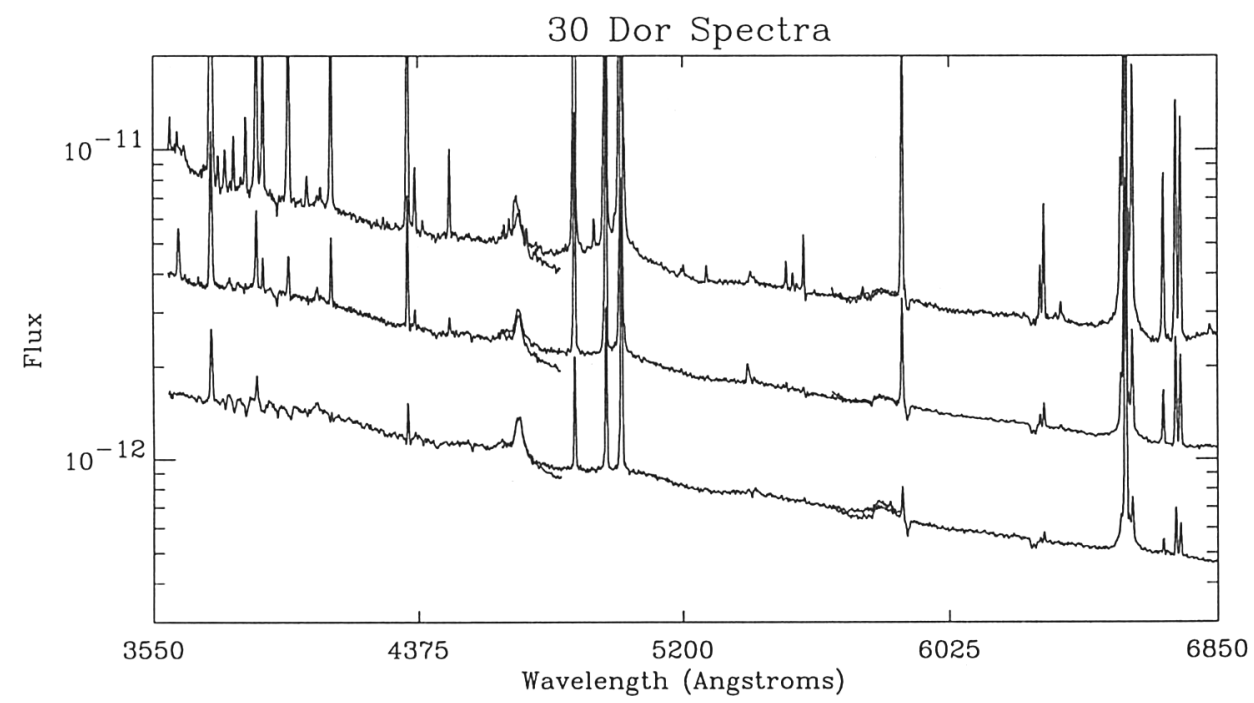

Figure 4. Optical spectrophotometry of the three square areas of Figure 3, corrected for extinction. Note the presence of broad emission lines due to He II at $\lambda 4686$ and C IV at $\lambda 5808$, and the relatively weak nebular lines in the smallest area.

ionized gas is outside the regions sampled. Typical slit widths used in optical spectrophotometry are $1.5^{\prime \prime}$. The $3^{\prime} \times 3^{\prime}$ size aperture illustrated in Figure 3 corresponds to this dimension in the LMC for an object at $6 \mathrm{Mpc}$. Thus a starburst with the luminosity and size of 30 Dor would just fill the slit at $6 \mathrm{Mpc}$. Geometric dilution, analogous to using slit widths equivalent to the projected smaller apertures of Figure 3, could be important since the starbursts are typically more luminous (and more extended) than 30 Dor.

\section{Conclusions}

For a (small) sample of WR galaxies there is good agreement between the observations of the equivalent widths of $\lambda 4686 \mathrm{He}$ II and $\mathrm{H} \beta$ and the model predictions. Detected WR galaxies have inferred ages that agree with the models. The non detection of WR features in some starburst galaxies (of ages between 3 to $6 \mathrm{Myr}$ ) could be due to continuum dilution (i.e., $\lambda 4686 \mathrm{He}$ II equivalent width below the detection limit). The $\mathrm{H} \beta$ inferred age will then be somewhat too large but this effect cannot be disentangled from the data. Geometric dilution or ionized gas area (volume) greater than the star area (volume) as illustrated by R 136/30 Dor might explain the few 'outlier' WR galaxies in the $\lambda 4686$ He II vs. $\mathrm{H} \beta$ equivalent width predictions. Alternatively, dust absorption or physical leakage of Lyc photons could be involved but these are difficult to quantify. One must adopt caution when using optical spectrophotometry of nebular emission lines to estimate hot star statistics. Imaging or spectrophotometry in the UV regions, which samples the hot stars directly, does not suffer from this problem (although other 
difficulties are present). Unfortunately, most of the quantitative work so far has utilized optical data.

WR galaxies are those starbursts with ages 3-6 Myr in which WR stars are present. In older bursts, these stars no longer exist; in younger systems, they have not yet appeared. I believe it would not be amiss to state what now appears to be obvious: WR phenomena in starburst galaxies are a normal part of evolution of young starbursts.

Acknowledgments. I appreciate hospitality from Drs. A. Herreo and C. Esteban at the Instituto de Astrofisica de Canarias where this paper was initiated. Support from the NSF is also appreciated. Dr. W. Vacca provided me with Figures 3 and 4, for which I am grateful and Dr. C. Leitherer comments on the manuscript for which I am obliged.

\section{References}

Allen, D.A., Wright, A.E., Goss, W.M. 1976, MNRAS 177, 91

Conti, P.S. 1991, ApJ 377, 115

Conti, P.S., Leitherer, C., Vacca, W.D. 1996, ApJ 461, L87

Contini, T. 1999, these Proceedings

Crowther, P.A., Beck, S.C., Willis, A.J., Conti, P.S., Morris, P.W., Sutherland, R.S. 1999, MNRAS in press

Johnson, K., Vacca, W.D., Leitherer, C., Conti, P.S. 1999, in preparation

Joseph, R.D. 1999, these Proceedings

Leitherer, C. 1999, these Proceedings

Leitherer, C. Heckman, T.M. 1995, ApJS 73, 1

Leitherer, C., Robert, C., Heckman, T.M. 1995, ApJS 99, 173

Leitherer, C., Vacca, W.D., Conti, P.S., Filippenko, A.V., Robert, C., Sargent, W.L.W. 1996, ApJ 465, 717

Schaerer, D. Vacca, W.D. 1998, ApJ 497, 618

Schaerer, D., Contini, T., Kunth, D. 1999, A\&A 341, 399

Schaerer, D. Contini, T., Pindao, M. 1999, A\&AS 136, 35

Schaerer, D. 1999, these Proceedings

Stasinska, G. 1999, these Proceedings

Vacca, W.D. 1994, ApJ 421, 140

Vacca, W.D., Conti, P.S. 1992, ApJ 401, 543

Vacca, W.D., Robert, C., Leitherer, C., Conti, P.S. 1995, ApJ 444, 647

Vacca, W.D., Garmany, C.D., Shull, M.J. 1996, ApJ 460, 914

\section{Discussion}

Heap: My comment concerns observing strategy. For years, people working on planetary nebulae obtained the nebular line-strength from narrow-band images (corrected for continuum emission). Since Kennicutt and others have made measurements of this kind, have you made use of them in your diagnostic plots?

Conti: My work, with Bill Vacca, used long-slit spectra only. I don't know of any quantitative analyses of $\mathrm{H} \alpha$ imaging of the WR galaxies I discussed, but it could be done. 
Lehmert: Can you comment on the relative distribution of where Wolf-Rayet features are seen in galaxies and how well do age estimates using diagnostics other than WR features agree with those determination made only using WR features?

Conti: My figure showed age estimates from the measured $\mathrm{H} \beta$ equivalent widths in a limited, but self-consistent, sample of WR galaxies from Vacca \& Conti (1992). The ages inferred for WR galaxies were all between 3-6 Myr, exactly as predicted by the models. The spatial distribution of WR features in galaxies follows the starburst locations.

R. Terlevich: Regarding theoretical predictions of the $W_{\mathrm{H} \beta}$, I would like to point out that there is a serious, and as far as I know, unsolved problem. While the models predict that $W_{\mathrm{H} \beta}$ during the first $3 \mathrm{Myr}$ is larger than $300 \AA$ and very probably around $500 \AA$, the largest $W_{\mathrm{H} \beta}$ observed in $\mathrm{H}$ II galaxies is $\sim 250 \AA$. This discrepancy probably indicates that either there is an underlying older population, increasing the optical continuum, or that there is a sourc of ionizing photons, about $50 \%$, or that dust is absorbing about half of the ionizing radiation, or a combination of the three effects. All this places serious doubts on the use of $W_{\mathrm{H} \beta}$ as an age estimator, unfortunately.

Conti: I agree that there exists a problem with the absence of high $\mathrm{H} \beta$ equivalent width starburst galaxies: so far none are observed. I don't think the problem is continuum dilution, as it would carry over into subsequent stages. It might be absorbtion of Lycphotons by the local dust. This effect would lessen as the burst ages and the dust is dissipated. This doesn't work for 30 Dor, but I suspect that the problem there is that we still have not obtained an aperature large enough to count all the $\mathrm{H} \alpha$ (Lyc) photons.

Hunter: I am concerned about the definition of Wolf-Rayet galaxies, since it depends on the signal-to-noise of one's spectrum. One could imagine that at sufficient signal-tonoise, all star-forming galaxies, including the Milky Way or the LMC, would have to be classified as WR galaxies.

Conti: With higher $\mathrm{S} / \mathrm{N}$, fainter emission lines may be detected, but there is a limit as the background continuum increases. I'd bet that in a spatially integrated spectrogram of the entire LMC, the WR stars, even those of R 136 would not be detectable. The same goes for IC 10: this galaxy is amenable to observation, spatially integrated; some one could do it.

Walborn: (1) Some complications may arise from internal spatial and temporal structures, combined with the viewing orientation, as shown by the two stage starbursts 30 Dor and N11. The nebular emission from 30 Dor is dominated by the curved filaments surrounding the semi-evacuated central region containing $\mathrm{R} 136$; beyond them are large molecular coulds, and there is extensive triggered star-formation at the interfaces. $\mathrm{N} 11$ is a giant shell with a Sco OB1-phase association in the evacuated core (including a WC!) and O3-type stars (but no WN yet!) in the surrounding $\mathrm{H}$ II regions. It is very fortunate that we have clear lines-of-sight to the cores of these three-dimensional structures in both cases; otherwise, we might be very confused.

(2) Could the starbursts with deficient WR features for those ages be more metaldeficient than the others?

Conti: (1) The spatially integrated spectrum of 30 Dor gives an age of 4-5 Myr. Clearly, this is a superposition of the two stage starburst. We are clearly mis-interpreting the real situation; yes one must be carefull.

(2) There is no correlation with a low $Z$ for those starburst galaxies of the age 3-6 Myr and no WR features. I suspect the problem to be continuum dilution. 